\title{
Addressing the feasibility of inboard direct-line injection of high-speed pellets, for core fueling of DEMO
}

\author{
A. Frattolillo $^{\mathrm{a}}$, L. R. Baylor ${ }^{\mathrm{b}}$, F. Bombarda ${ }^{\mathrm{a}}$, F. Cismondi ${ }^{\mathrm{c}}$, A. Colangeli ${ }^{\mathrm{a}}$, S. K. Combs ${ }^{\mathrm{b}}$, Chr. \\ Day $^{\mathrm{d}}$, G. D’Elia ${ }^{\mathrm{a}}$, T. E. Gebhart ${ }^{\mathrm{b}}$, F. Iannone ${ }^{\mathrm{a}}$, P.T. Lang ${ }^{\mathrm{e}}$, S. J. Meitner ${ }^{\mathrm{b}}$, S. Migliori ${ }^{\mathrm{a}}$, F. Moro ${ }^{\mathrm{a}}$, \\ R. Mozzillo ${ }^{f}$, B. Pégourié ${ }^{\mathrm{g}}$, B. Ploeckl ${ }^{\mathrm{e}}, \mathrm{S}$. Podda ${ }^{\mathrm{a}}$, F. Poggi ${ }^{\mathrm{a}}$ \\ ${ }^{a}$ ENEA C.R. Frascati, 00044 Frascati, Rome, Italy \\ ${ }^{b}$ Oak Ridge National Laboratory, Oak Ridge (TN), U.S.A. \\ ${ }^{c}$ EUROfusion Consortium, Programme Management Unit, Garching, Germany \\ ${ }^{d}$ Karlsruhe Institute of Technology, 76021 Karlsruhe, Germany \\ ${ }^{e}$ Max Planck Institute for Plasma Physics, 85748 Garching, Germany \\ ${ }^{f}$ Consorzio CREATE, Naples, Italy \\ ${ }^{g}$ CEA-IRFM, 13108 Saint-Paul-lez-Durance, France
}

\begin{abstract}
Pellet injection represents, to date, the most promising option for core fuelling of the EU-DEMO tokamak. Simulations with the HPI2 pellet ablation/deposition code indicate, however, that sufficiently deep fuel deposition requires injection from the High Field Side (HFS) at velocities $\gtrsim 1 \mathrm{~km} / \mathrm{s}$. Two complementary inboard injection schemes are being explored: one makes use of guide tubes with curvature radii $\geq 6 \mathrm{~m}$ in the attempt of preserving pellet integrity at speeds of $\sim 1 \mathrm{~km} / \mathrm{s}$; the other is investigating the feasibility of injecting high-speed $(\sim 3 \mathrm{~km} / \mathrm{s})$ pellets along "direct line of sight" (DLS) trajectories, from either the HFS or a vertical port. Options using quasivertical DLS paths routed across the upper vertical port have been explored first, as they can be more easily integrated; Unfortunately, the radial position of the available vertical access $(\gtrsim 9 \mathrm{~m}$ from the machine axis) turns out to be unfavorable; further simulations with the HPI2 code predict indeed that vertical injection may be effective only if pellets trajectories are well inboard the magnetic axis. High-speed injection through oblique inboard "DLS" paths, not interfering with the Central Solenoid (CS), are instead predicted to yield good performance, provided that the injection location is $\lesssim 2.5 \mathrm{~m}$ from the equatorial mid-plane. The angular spread of high-speed free-flight pellets, recently measured using an existing facility, turns out to be enclosed within $\sim 0.7^{\circ}$. This scatter cone may require significant cut off volume of the Breeding Blanket (BB). Moreover, DLS in-vessel conical penetrations may increase the neutron flux outside of the bio-shield, and also result in a significant heat load in the cryogenic pellet source.These issues are being investigated, to identify suitable shielding strategies; preliminary results are reported. The suitability of straight guide tubes to reduce the scatter cone, and hence the corresponding open cross section on BB penetration and the neutron streaming, will be explored as a further step.
\end{abstract}

Keywords: EU-DEMO tokamak, High Field Side high-speed pellet injection, straight guide tubes.

\section{Introduction}

Pellet injection still represents, to date, the prime candidate option for core fuelling of the EU-DEMO (DEMOnstration fusion reactor) tokamak [1]. Pellet penetration and fuel deposition profiles have been simulated, for different injection locations, using the HPI2 pellet ablation-deposition code [2], assuming specific EU-DEMO $2017 \mathrm{H}$-mode plasma scenarios and the ITER reference pellet mass $\left(6 \times 10^{21}\right.$ atoms $)$. The results indicate that injection from the Low Field Side (LFS) is ineffective, regardless of the pellet speed, whereas efficient fuelling can be achieved launching pellets from the High Field Side (HFS) at velocities $\gtrsim 1$ $\mathrm{km} / \mathrm{s}$ [3]. To implement suitable inboard injection schemes for the EU-DEMO, two complementary approaches are being considered [4]: one is aimed at improving the design of guide tubes, using curvature radii $\geq 6 \mathrm{~m}$ in the attempt to preserve pellet integrity at $1 \mathrm{~km} / \mathrm{s}$; the other is investigating the feasibility of injecting high-speed $(\sim 3 \mathrm{~km} / \mathrm{s})$ pellets along "direct line of sight" (DLS) trajectories, from either the HFS or a vertical port. The identification and integration of suitable oblique HFS straight injection paths, compatible with existing constraints and, in particular, avoiding interferences with the central solenoid (CS), requires careful investigation. Options using the upper vertical port have therefore been explored first, since they can be more easily integrated; the results of this analysis are reported in section 2. The angular spread of the trajectories of high-speed free-flight pellets, and/or the suitability of straight guide tubes to reduce the scatter cone and the corresponding open cross section on breeding blanket (BB) penetration, are being explored using a recently upgraded existing facility (as shortly described in section 3). As a first step, the scatter cone of high-speed free-flight pellets has been measured, as reported in section 4. The neutron flux across DLS injection paths is also being investigated to identify suitable shielding strategies; the results of preliminary analyses, using conically shaped in-vessel penetrations routed across the upper vertical port and compatible with the measured scatter cone, are presented in section 5. 


\section{Identification of DLS injection paths using the vertical upper port}

The EU-DEMO design includes a large vertical upper port for Remote Maintenance (RM) [5], whose access is at the level of the bio-shield top lid $(12.78 \mathrm{~m}$ above the VV entrance and $18 \mathrm{~m}$ above the equatorial mid-plane). The possibility of routing DLS paths across a rectangular shaped $(1.5 \mathrm{~m} \times 0.7 \mathrm{~m})$ free duct, located inside this vertical port at a distance $\geq 9 \mathrm{~m}$ from the machine axis (figure 1), has been investigated.

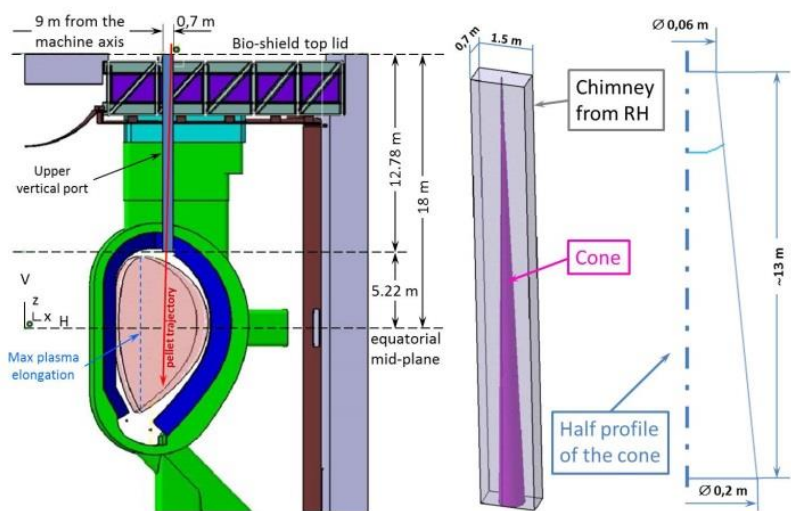

Fig. 1. Schematics of EU-DEMO upper vertical port

Unfortunately, fuel deposition simulations performed using the HPI2 code predict that vertical injection may be effective, to some extent, only if pellets are injected from radial positions well inboard the magnetic axis of the plasma [6], so that DLS injection paths routed across this vertical duct are expected to result in very poor fueling efficiency. High-speed injection through oblique inboard "DLS" paths, not interfering with the CS, are instead predicted to yield good fueling performance, provided that the trajectories intercept the separatrix at a distance from the equatorial mid-plane $\lesssim 2.5 \mathrm{~m}$ [6]. Their integration in the design of DEMO need therefore to be investigated.

\section{Recent upgrading of the ENEA-ORNL facility}

The high-speed four-barrel pellet injector prototype used for this study [7-12] is located at ORNL (figure 2): it can be operated either locally or, via the internet, from a remote control station at ENEA Frascati. This facility has been recently upgraded in order to improve the quality of deuterium ice and produce pellets capable of

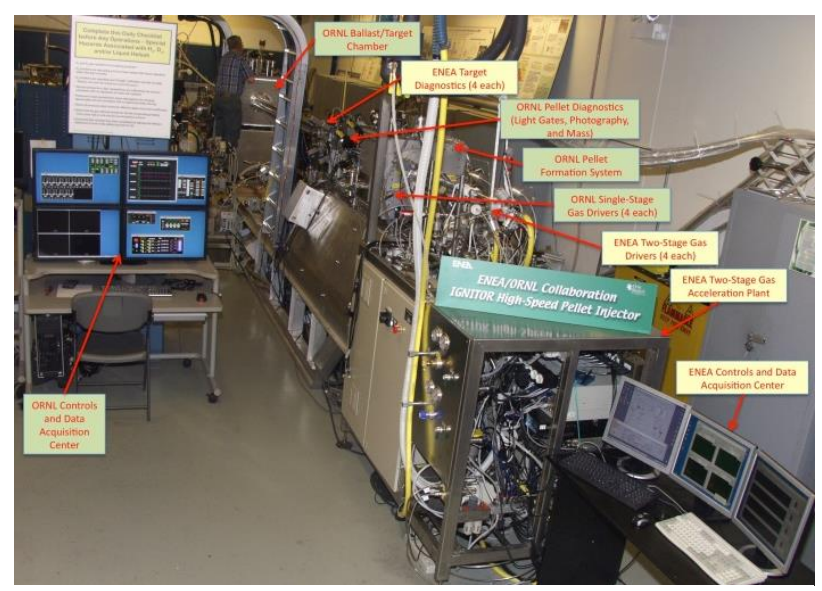

Fig. 2. The high-speed four-barrel pellet injector at ORNL withstanding the huge stress required to accelerate them at speeds in excess of $2 \mathrm{~km} / \mathrm{s}$, without fracturing. The existing two-stage pulse tube cryo-generator (Cryomech PT810, cooling power of $80 \mathrm{~W} @ 80 \mathrm{~K}$ on the first stage and14 W@20K on the second), used to cool down the cryostat during initial tests, has been replaced by a similar but more powerful unit (Cryomech PT415, featuring a refrigerating power of $40 \mathrm{~W} @ 45 \mathrm{~K}$ on the first stage and 1.5 W@4.2 K on the second, with a nominal minimum temperature of $\sim 2.8 \mathrm{~K}$ ). Cooling tests with the PT415 have shown a significant drop of the temperatures achieved at both the radiation shield (from $\sim 100 \mathrm{~K}$ down to $\sim 54 \mathrm{~K}$ ) and the freezing region (from $\sim 10 \mathrm{~K}$ to $\sim 8 \mathrm{~K}$ ), with a consistent reduction of the overall time required to cool down the cryostat [12]. However, the temperature measured at the second cooling stage $(\sim 5 \mathrm{~K})$ turns out to be $\sim 3 \mathrm{~K}$ lower than that achieved by the freezing zone, suggesting that the latter is prevented from getting even colder because of an excessive heat load, perhaps due to thermal conduction along the barrels. To substantiate this analysis, the diode sensors of barrels 2 and 3 have been relocated to measure the temperatures at about $2.5 \mathrm{~cm}$ upstream (breech side) and downstream (muzzle side) of the freezing zone of barrel 4; as a matter of fact, temperature as high as $\sim 200 \mathrm{~K}$ and $\sim 146 \mathrm{~K}$ have been measured respectively, thus validating our hypothesis. The cryostat configuration has therefore been modified, adding thermal shorts on both the breech and the muzzle sides of the freezing zone, to redirect the incoming heat flow toward the $1^{\text {st }}$ stage of the cryo-cooler (figure 3 ).

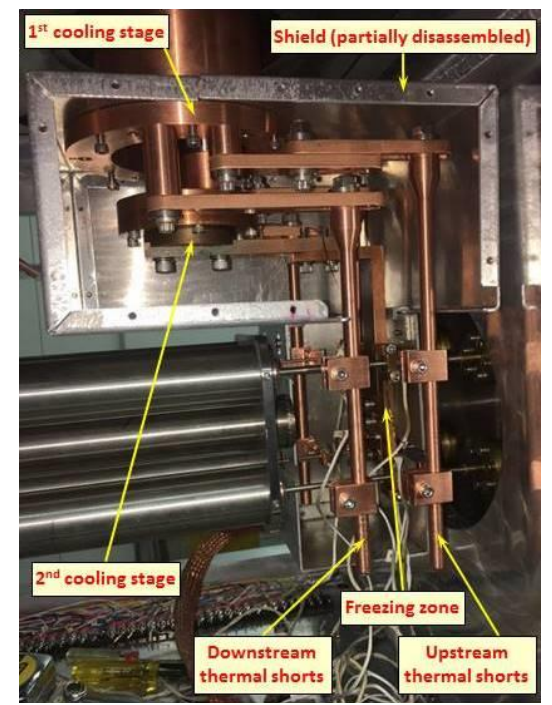

Fig. 3. The new cryostat configuration with added upstream and downstream thermal links to the first cooling stage

With this new barrel arrangement (and the addition of multilayer insulation on the radiation shield), the temperatures measured both upstream and downstream of the freezing zone are drastically reduced (to $\sim 40 \mathrm{~K}$ ), while those measured at the second-stage cold head and at the freezing zone can reach steady state values as low as $4.2 \mathrm{~K}$ and $5.7 \mathrm{~K}$ respectively, thus allowing very stable control of the freezing zone temperature during pellet formation, and removing the unwanted oscillation that are usually observed when the desired temperature 
set point approaches the lowest temperature that the system can achieve.

After performing several remote operation sessions, to optimize pellet formation parameters with this new configuration, a joint experimental campaign at ORNL was carried out in December 2016, that demonstrated improved injector performance, with increased launching speeds of intact pellets from $\sim 2.0 \mathrm{~km} / \mathrm{s}$ up to $\sim 2.4 \mathrm{~km} / \mathrm{s}$.

\section{Dispersion data of high-speed free-flight pellets}

In order to ensure free-flight of the pellets from the gun muzzle to the final target, placed $3772 \mathrm{~mm}$ downstream, the microwave cavity has been removed, while a single large-diameter $(\varnothing 150 \mathrm{~mm})$ tube has been used to connect the injector to the final target tank, as shown in figure 4. A further short joint experimental

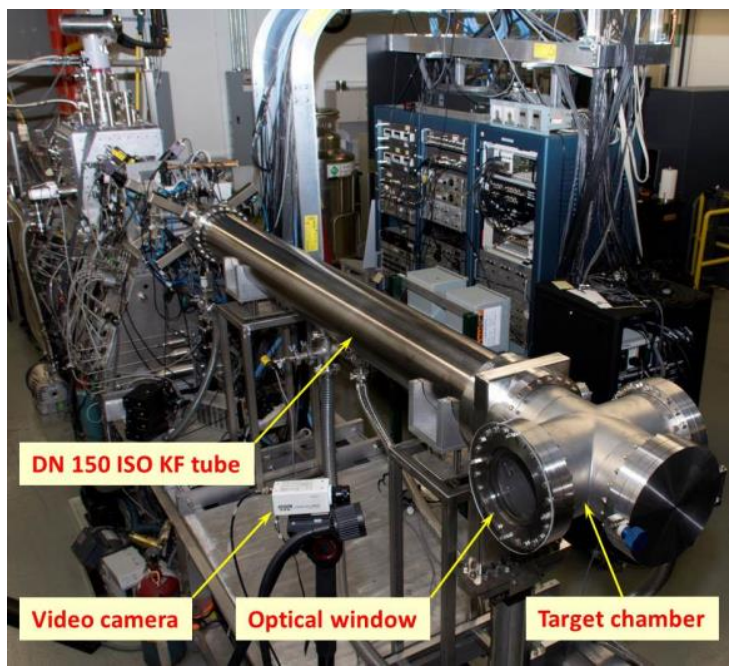

Fig. 4. The new IPI arrangement ensuring pellet's free-flight

campaign has been carried out at ORNL in July 2018, using only the largest bore barrel $(4.4 \mathrm{~mm})$; this pellet size has been selected since it more closely approaches that required for DEMO core fueling. After preliminary testing the new injector configuration at low speeds $(\lesssim 1$ $\mathrm{km} / \mathrm{s}$ ), using ORNL propellant valves, high-speed tests using the ENEA TSG have been finally carried out. The aluminum targets were replaced frequently (no more than four shots with the same target), to facilitate the subsequent analysis. Moreover, the impact of each pellet on the target has been monitored by means of a video camera, providing an easy way to record a short video of each impact and to capture an image of the target after every single shot. This has turned out to be a very useful tool to precisely identify the imprint of each pellet. A total of 62 pellets were launched at speed ranging from 1.4 up to $2.44 \mathrm{~km} / \mathrm{s}$. The resulting overall impact pattern is shown in figure 5, where the intersection of the red dashed lines identify the projection of the four barrel's axes. Black and red dots refer to intact pellets travelling at different speeds; the three empty dots represent the imprints of the main piece of high-speed pellets associated with a couple of much smaller fragments (as witnessed by their traces on the target). The (x,y) coordinates of each point have been measured with respect to the reference system shown in fig. 5, and the two sets of values (assumed to be statistically

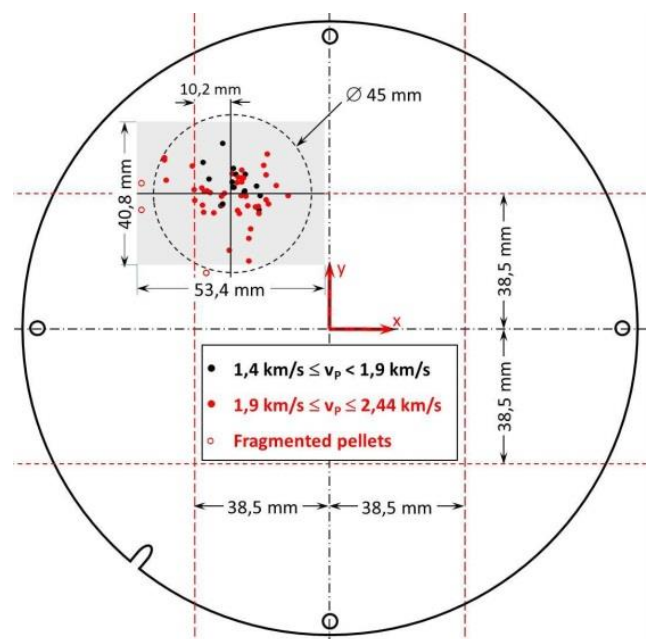

Fig. 5. Impact pattern of free-flight high-speed pellets

independent) have been separately fitted using a normal distribution, in order to identify the center of the impact pattern on the target, given by the average values $\left(\mathrm{x}_{0}\right.$, $\mathrm{y}_{0}$ ), and the scatter cone width, associated with the standard deviations of the two normal distributions. The results are reported in table 1.

Table 1. normal distribution parameters of impact points

\begin{tabular}{cccc}
\hline $\mathrm{x}_{0}(\mathrm{~mm})$ & $\mathrm{y}_{0}(\mathrm{~mm})$ & $\sigma_{\mathrm{x}}(\mathrm{mm})$ & $\sigma_{\mathrm{y}}(\mathrm{mm})$ \\
\hline-28.30 & 38.47 & 8.9 & 6.8 \\
\hline
\end{tabular}

This analysis indicates that the injector is pointing about $10.2 \mathrm{~mm}$ to the right, as compared to the expected aiming position. About $99.7 \%$ of values drawn from a normal distribution are within three standard deviations. This means that $99.7 \%$ of the pellets are expected to hit the target within a distance of about $\pm 26.7 \mathrm{~mm}$ from $\mathrm{x}_{0}$ and about $\pm 20.4 \mathrm{~mm}$ from $\mathrm{y}_{0}$, as shown by the shadowed area in fig. 5. This figure also shows that, except for the three fragmented pellets, all the impacts fall within a circle of $\approx 45 \mathrm{~mm}$ in diameter, centered in $\left(\mathrm{x}_{0}, \mathrm{y}_{0}\right)$. If one takes into account the distance of the target from the gun muzzle, the angular scattering of intact pellets trajectories turns out to be about $0.7^{\circ}$ $(0.012 \mathrm{rad})$, which is the same value that had been previously measured for this barrel at speeds of $\sim 1$ $\mathrm{km} / \mathrm{s}$, using the ORNL propellant valve [13]. Moreover, this angular spread is not far from that measured $\left(0.57^{\circ}\right)$ many years ago using the high-speed multiple-barrels pellet injectors for the Frascati Tokamak Upgrade (FTU) [14].

\section{The neutron flux across DLS injection paths}

A preliminary assessment of the neutron flux through a vertical DLS injection path has been performed using the Monte Carlo N-Particle (MCNP) code [15] and the JEFF 3.2 nuclear data libraries [16]. The free flight fueling assembly with conical aperture (as shown in figure 1) has been defined in a $20^{\circ}$ DEMO model integrating the WCLL (Water Cooled Lithium Lead) Single-Module-Segment breeding blanket [17]. As a first step, the neutron flux has been calculated for two different configurations:

- Free flight injection line completely open 


\section{- $\quad$ Free flight injection line with VV-like plug}

In both cases, calculation has been carried out for two locations along the injection path: 1) outside the VV, 7.3 $\mathrm{m}$ from the equatorial mid-plane, and 2) above the external surface of the Bio-shield, at $18.5 \mathrm{~m}$ from the equatorial mid-plane (figure 6).

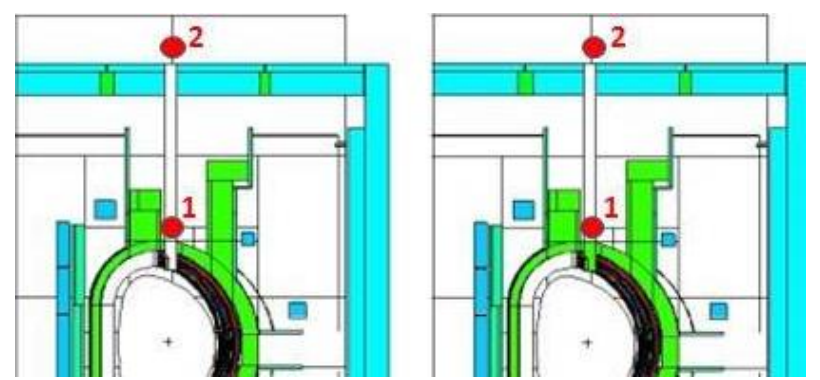

Fig. 6. Poloidal section of the MCNP DEMO model with chimney completely open (left panel), model with VV-like plug (right panel). Red dots specify the scoring positions.

The neutron fluxes estimated with the open injection line are $5.05 \times 10^{12}$ and $7.28 \times 10^{10} \mathrm{n} / \mathrm{cm}^{2} / \mathrm{s}$ respectively. The integration of the VV-like plug ensures a substantial reduction of the scattered neutron flux across the conical penetration $(\varnothing 200 \mathrm{~mm})$ : respectively $6.01 \times 10^{9}$ and $9.15 \times 10^{8} \mathrm{n} / \mathrm{cm}^{2} / \mathrm{s}$ outside the VV and the Bio-shield. This preliminary assessment highlights the necessity to develop a dedicated shielding strategy to further reduce the radiation streaming along the free flight injection path, such as introducing an additional upper plug. If the use of straight guide-tubes will reveal a suitable option, the resulting neutron flux is expected to further decrease.

\section{Conclusions}

HFS injection of high-speed pellets, along either oblique or vertical DLS trajectories, is being studied as a potential method to achieve core fuelling of the EUDEMO tokamak. The main issues to be dealt with, are those of evaluating, and possibly minimizing, the aperture across the $\mathrm{BB}$ required for penetration of the injection line, as well as the neutron flux through such DLS in-vessel penetrations. The scatter cone of freeflight high-speed (up to $2.4 \mathrm{~km} / \mathrm{s}$ ) pellets has been recently measured; it has been found to be within $0.7^{\circ}$. As a first approach, calculation are also being performed, using the MCNP code, to estimate the neutron flux across a conical aperture (compatible with the measured dispersion angle) routed inside the vertical upper port, with the aim of identifying suitable shielding strategies. Preliminary results indicate that the addition of a plug at the $\mathrm{VV}$ end of the cone reduces the radiation streaming along the free flight injection path, by two orders of magnitude. The introduction of an additional upper plug is presently being investigated, to assess whether a further significant reduction of the neutron flux may be achieved. As a next step, the feasibility of using straight guide tubes to minimize both the aperture across the $\mathrm{BB}$ and the neutron flux will be investigated.

\section{Acknowledgments}

This work has been carried out within the framework of the EUROfusion Consortium and has received funding from the Euratom research and training programme 2014-2018 under grant agreement No 633053. The views and opinions expressed herein do not necessarily reflect those of the European Commission.

\section{References}

[1] Chr. Day et al., Consequences of the technology survey and gap analysis on the EUDEMO R\&D programme in tritium, matter injection and vacuum, Fus. Eng. Design 109-111 (2016) 299-308

[2] B. Pégourié et al., Modelling of pellet ablation in additionally heated plasmas, Plasma Phys. Control. Fusion 47 (2005) 17-35.

[3] B. Pégourié et al., Physical constraints on the design of the DEMO pellet fueling system, $43^{\text {rd }}$ EPS Conf. Plasma Phys, (2016), contribution P4.076.

[4] A. Frattolillo et al., An innovative approach for DEMO core fuelling by inboard injection of high-speed pellets, Fus. Eng. Design 124 (2017) 846-849

[5] C. Vorpahl et al., Initial Configuration studies of the upper vertical port of the European DEMO, this Conf. (P3.137)

[6] A. Frattolillo et al., Core Fueling of DEMO by Direct Line Injection of High-Speed Pellets From the HFS, IEEE Trans. Plasma Sci. 46(5) (2018) 1429-1435

[7] S. Migliori et al., The Compact, Multiple Barrel High Speed Pellet Injector for the Ignitor Experiment, Proc. of the 21st IEEE/NPSS Symposium on Fusion Engineering (SOFE05), Knoxville (TN), U.S.A., Sept. 26-29 2005

[8] A. Frattolillo et al., Peaked density profiles and high speed pellet injection for the Ignitor burning plasma experiment, Proc. of the $33^{\text {rd }}$ EPS Conference on Plasma Phys. Rome, Italy, 19 - 23 June 2006, ECA Vol. 301, P4.185 (2006)

[9] A. Frattolillo et al., Advances On The High Speed Ignitor Pellet Injector (IPI), Proc. of the $24^{\text {th }}$ IEEE/NPSS Symposium On Fusion Engineering (SOFE2011), Chicago (IL), June 26-31, 2011

[10] A. Frattolillo et al., The Compact, Four Barrel High Speed Pellet Injector For The Ignitor Experiment, Proc. of the $22^{\text {nd }}$ IEEE/NPSS Symposium on Fusion Engineering (SOFE07), Albuquerque (NM), U.S.A., June 17-21 2007

[11] F. Bombarda et al., Fusion Engineering and Design 124 (2017) 797-800

[12] A. Frattolillo et al., Progress with the ENEA-ORNL highspeed four barrels pellet injector, Proc. of the $42^{\text {nd }}$ EPS Conference on Plasma Physics (2015), P2.155; see also the poster by G. D'Elia et al., in this Conference

[13] S. K. Combs, private communication.

[14] A. Frattolillo et al., High-speed multishot pellet injector prototype for the Frascati Tokamak Upgrade, Rev. Sci. Instrum. Vol. 69 (7), July 1998, p. 2675-2680.

[15] X-5 Monte Carlo Team: MCNP - A General Monte Carlo N-Particle Transport Code, Version 5, LANL, Los Alamos, New Mexico, USA, April 2003.

[16] JEFF3.2 nuclear data library, http://www.oecdnea.org/dbforms/data/eva/evatapes/jeff_32/.

[17] F. Moro et al., Neutronic analyses in support of the WCLL DEMO design development, Fus. Eng. Design, (2018), in press, doi: 10.1016/j.fusengdes.2018.04.113. 